\title{
A dynamical study of the chirally rotated Schrödinger functional in QCD
}

\author{
Mattia Dalla Brida* \\ School of Mathematics, Trinity College Dublin, Dublin 2, Ireland, \\ NIC, DESY, Platanenallee 6, 15738 Zeuthen, Germany \\ E-mail: mattia@maths.tcd.ie

\section{Stefan Sint} \\ School of Mathematics, Trinity College Dublin, Dublin 2, Ireland, \\ NIC, DESY, Platanenallee 6, 15738 Zeuthen, Germany \\ E-mail: sint@maths.tcd.ie
}

\begin{abstract}
The chirally rotated Schrödinger functional for Wilson-fermions allows for finite-volume, massindependent renormalization schemes compatible with automatic $\mathrm{O}(a)$ improvement. So far, in QCD, the setup has only been studied in the quenched approximation. Here we present first results for $N_{\mathrm{f}}=2$ dynamical quark-flavours for several renormalization factors of quark-bilinears. We discuss how these renormalization factors can be easily obtained from simple ratios of twopoint functions, and show how automatic $\mathrm{O}(a)$ improvement is at work. As a by-product of this investigation the renormalization of the non-singlet axial current, $Z_{A}$, is determined very precisely.
\end{abstract}

DESY $14-247$

The 32nd International Symposium on Lattice Field Theory

23-28 June, 2014

Columbia University New York, $N Y$

\footnotetext{
*Speaker.
} 


\section{Introduction}

The Schrödinger functional (SF) is a powerful tool to solve non-perturbative renormalization problems in lattice QCD $[1,2]$. The SF, allows for the definition of gauge-invariant, massindependent, finite-volume renormalization schemes, which are suitable for both non-perturbative and perturbative evaluations. The standard lattice formulation of the SF, however, is in conflict with the argument of automatic $\mathrm{O}(a)$ improvement of massless Wilson-fermions in finite volume [3]. The reason is that the SF boundary conditions $\left(P_{ \pm}=\frac{1}{2}\left(1 \pm \gamma_{0}\right)\right)$,

$$
\left.P_{+} \psi(x)\right|_{x_{0}=0}=\left.\bar{\psi}(x) P_{-}\right|_{x_{0}=0}=0,\left.\quad P_{-} \psi(x)\right|_{x_{0}=T}=\left.\bar{\psi}(x) P_{+}\right|_{x_{0}=T}=0,
$$

explicitly break chiral symmetry, and therefore the aforementioned argument cannot go through.

In $[4,5]$, it has been shown that automatic $\mathrm{O}(a)$ improvement can be rescued for an even number of quark-flavours by changing the boundary conditions for the fields. The basic idea is to extend the principles of twisted-mass lattice QCD [6] to the SF. More precisely, given the isospin doublets $\psi$ and $\bar{\psi}$ satisfying the standard SF boundary conditions (1.1), one considers the chiral rotation,

$$
\psi \equiv R \chi \equiv e^{i \frac{\pi}{2} \gamma_{5} \frac{\tau^{3}}{2}} \chi, \quad \bar{\psi} \equiv \bar{\chi} R \equiv \bar{\chi} e^{i \frac{\pi}{2} \gamma_{5} \frac{\tau^{3}}{2}}
$$

The fields $\chi$ and $\bar{\chi}$ so defined satisfy the chirally rotated SF ( $\chi \mathrm{SF})$ boundary conditions,

$$
\left.\tilde{Q}_{+} \chi(x)\right|_{x_{0}=0}=\left.\bar{\chi}(x) \tilde{Q}_{+}\right|_{x_{0}=0}=0,\left.\quad \tilde{Q}_{-} \chi(x)\right|_{x_{0}=T}=\left.\bar{\chi}(x) \tilde{Q}_{-}\right|_{x_{0}=T}=0,
$$

where $\tilde{Q}_{ \pm} \equiv \frac{1}{2}\left(1 \pm i \gamma_{0} \gamma_{5} \tau^{3}\right)$, and $\tau^{1,2,3}$ are Pauli matrices. Using the invariance of these boundary conditions w.r.t. the field transformation,

$$
P_{5}: \quad \chi(x) \rightarrow i \gamma_{0} \gamma_{5} \tau^{3} \chi(\tilde{x}), \quad \bar{\chi}(x) \rightarrow-i \bar{\chi}(\tilde{x}) \gamma_{0} \gamma_{5} \tau^{3}, \quad \tilde{x}=\left(x_{0},-\mathbf{x}\right),
$$

i.e. $\left[\tilde{Q}_{ \pm}, \gamma_{0} \gamma_{5} \tau^{3}\right]=0$, automatic $\mathrm{O}(a)$ improvement can be recovered [5]. In addition, as the chiral field rotation is a non-anomalous symmetry of the continuum massless QCD action, one can derive universality relations between standard SF and $\chi \mathrm{SF}$ correlation functions, of the form,

$$
\langle O[\psi, \bar{\psi}]\rangle=\langle O[R \chi, \bar{\chi} R]\rangle .
$$

On the lattice with Wilson-fermions, the above relations are then expected to hold among properly renormalized correlation functions up to discretization effects.

The realization of the $\chi \mathrm{SF}$ boundary conditions (1.3) with Wilson-fermions is non-trivial, since it requires the non-perturbative renormalization of a boundary counterterm [5]. The presence of this counterterm is a direct consequence of the explicit breaking of flavour and parity symmetry by the regularization. The corresponding coefficient $z_{f}\left(g_{0}\right)$ is thus finite, and can be fixed by imposing parity/flavour symmetry restoration on a given observable. Once $z_{f}\left(g_{0}\right)$ is determined and the quark-masses are set to zero, automatic $\mathrm{O}(a)$ improvement is at work. This means that all bulk $\mathrm{O}(a)$ effects are located in $P_{5}$-odd correlation functions, while $P_{5}$-even observables are free from these contributions. Note however that $\mathrm{O}(a)$ lattice artifacts are in general not absent from $P_{5}$ even quantities, since the SF boundary conditions introduce additional discretization effects which are not taken care of by the argument of automatic $\mathrm{O}(a)$ improvement. On the other hand, these effects can be eliminated by adjusting a couple of $\mathrm{O}(a)$ boundary counterterms in the action [5]. 
So far, the $\chi$ SF has only been studied systematically in the context of perturbation theory $[7,8]$, and in the quenched approximation $[9,10,11]$. These studies confirm the validity of the universality relations (1.5) in the continuum limit, and the realization of automatic $\mathrm{O}(a)$ improvement as described above. Following these developments, in this contribution we present first results from dynamical simulations of $N_{\mathrm{f}}=2 \mathrm{O}(a)$-improved massless Wilson-fermions with $\chi$ SF boundary conditions. More precisely, expanding on the ideas presented in [9], in Section 2 we discuss how the universality relations (1.5) can be exploited for an efficient computation of several finite renormalization constants of interest. After a short description of the lattice set-up in Section 3, results for the renormalization of the non-singlet vector and axial currents are then presented in Section 4 . These determinations together with the study of several $P_{5}$-odd correlators, also provide a non-trivial test for automatic $\mathrm{O}(a)$ improvement, as discussed in Section 5. Finally, in Section 6 we present some results for the renormalization of the pseudo-scalar density.

\section{Renormalization conditions from universality relations}

As a starting point, we consider the standard SF correlation functions defined by [12],

$$
f_{X}\left(x_{0}\right)=-\frac{1}{2}\left\langle X^{f_{1} f_{2}}(x) \mathscr{O}_{5}^{f_{2} f_{1}}\right\rangle, \quad k_{Y}\left(x_{0}\right)=-\frac{1}{6} \sum_{k=1}^{3}\left\langle Y_{k}^{f_{1} f_{2}}(x) \mathscr{O}_{k}^{f_{2} f_{1}}\right\rangle, \quad f_{1}=-\frac{1}{2}\left\langle\mathscr{O}_{5}^{f_{1} f_{2}} \mathscr{O}_{5}^{\prime f_{2} f_{1}}\right\rangle .
$$

Here the fields $X$ and $Y_{k}$ stand for the quark-bilinears, $X=A_{0}, V_{0}, S, P$, and $Y_{k}=A_{k}, V_{k}, T_{k 0}, \widetilde{T}_{k 0}$, defined as usual e.g. $A_{\mu}^{f_{1} f_{2}}=\bar{\psi}_{f_{1}} \gamma_{\mu} \gamma_{5} \psi_{f_{2}}$, while the fields $\mathscr{O}_{5, k}$ and $\mathscr{O}_{5}^{\prime}$, are bilinears of non-Dirichlet quark-field components located near the boundaries of the lattice. Note that in the following we imagine a set-up with 2 up- and 2 down-type valence quarks, i.e. $f_{1}, f_{2}=u, u^{\prime}, d, d^{\prime}$ [9].

Given the SF correlation functions (2.1), through the chiral rotation (1.2) one can easily derive universality relations among the corresponding correlation functions in the $\chi \mathrm{SF}$ (cf. (1.5)). The latter will be denoted by $g_{X}^{f_{1} f_{2}}, l_{Y}^{f_{1} f_{2}}$, and $g_{1}^{f_{1} f_{2}}$. For example, the following universality relations among $P_{5}$-even correlators can be obtained [9]:

$$
f_{A}=g_{A}^{u u^{\prime}}=-i g_{V}^{u d}, \quad f_{P}=i g_{S}^{u u^{\prime}}=g_{P}^{u d}, \quad k_{V}=l_{V}^{u u^{\prime}}=-i l_{A}^{u d}, \quad f_{1}=g_{1}^{u u^{\prime}}=g_{1}^{u d} .
$$

Similar relations can be worked out for $P_{5}$-odd correlators [9]:

$$
f_{V}=g_{V}^{u u^{\prime}}=-i g_{A}^{u d}, \quad f_{S}=i g_{P}^{u u^{\prime}}=g_{S}^{u d}, \quad k_{A}=l_{A}^{u u^{\prime}}=-i l_{V}^{u d}, \quad k_{\widetilde{T}}=i l_{T}^{u u^{\prime}}=l_{\widetilde{T}}^{u d} .
$$

As already mentioned, universality relations such as (2.2) are expected to hold among properly renormalized lattice correlation functions up to discretization effects. As an example consider the first relation in (2.2), one then expects: $\left(g_{A}^{u u^{\prime}}\right)_{R}=\left(-i g_{\widetilde{V}}^{u d}\right)_{R}+\mathrm{O}\left(a^{2}\right) \Rightarrow Z_{A} g_{A}^{u u^{\prime}}=-i g_{\widetilde{V}}^{u d}+\mathrm{O}\left(a^{2}\right)$, where $\widetilde{V}_{\mu}$ is the (conserved) point-split vector current and $Z_{A}$ the axial current renormalization constant. In fact, one can turn the tables, and impose the validity of a set of universality relations at finite lattice spacing in order to define the finite renormalization constants of interest. In particular, given the relations (2.2) one can define,

$$
\left.Z_{A}^{g} \equiv \frac{-i g_{\widetilde{V}}^{u d}\left(x_{0}\right)}{g_{A}^{u u^{\prime}}\left(x_{0}\right)}\right|_{x_{0}=\frac{T}{2}},\left.\quad Z_{A}^{l} \equiv \frac{i l_{\widetilde{V}}^{u u^{\prime}}\left(x_{0}\right)}{l_{A}^{u d}\left(x_{0}\right)}\right|_{x_{0}=\frac{T}{2}},\left.\quad Z_{V}^{g} \equiv \frac{g_{\widetilde{V}}^{u d}\left(x_{0}\right)}{g_{V}^{u d}\left(x_{0}\right)}\right|_{x_{0}=\frac{T}{2}},\left.\quad Z_{V}^{l} \equiv \frac{l_{\widetilde{V}}^{u u^{\prime}}\left(x_{0}\right)}{l_{V}^{u u^{\prime}}\left(x_{0}\right)}\right|_{x_{0}=\frac{T}{2}}
$$


Likewise for the ratio $Z_{P} / Z_{S}$, for example. To conclude, we remark that these definitions are $\mathrm{O}(a)$ improved. First of all, the renormalization constants are obtained from $P_{5}$-even correlation functions, hence no bulk $\mathrm{O}(a)$ counterterms are needed for their $\mathrm{O}(a)$ improvement. Secondly, the $\mathrm{O}(a)$ boundary counterterm contributions to the correlation functions in (2.4) cancel out in the ratios.

\section{Lattice set-up}

In this work we consider $N_{\mathrm{f}}=2 \mathrm{O}(a)$-improved Wilson-quarks with $\chi \mathrm{SF}$ boundary conditions. The specific fermionic action that we consider is the one described in $[8,9]$. The gauge action is also taken to be Wilson's [1], where we set the boundary gauge fields to zero. The lattice geometry is then specified by the condition $T=L$, where $L$ is the lattice spatial extent. Lastly, the $\mathrm{O}(a)$ boundary counterterm coefficients are set to their 1-loop perturbative values taken from $[7,8]$.

Given the details of the $\chi$ SF set-up, the renormalization constants (2.4) are now completely specified by choosing the renormalization conditions for the bare parameters. Specifically, we set the bare quark-mass to its critical value $m_{\mathrm{cr}}$ by requiring the PCAC mass, $m_{\mathrm{PCAC}} \propto \partial_{0} g_{A}^{u d}(T / 2)$, to vanish. The boundary counterterm coefficient $z_{f}$ instead, is fixed by imposing the $P_{5}$-odd correlator $g_{A}^{u d}(T / 2)$ to be zero. Note that $m_{\mathrm{cr}}$ and $z_{f}$ are defined by these conditions only up to $\mathrm{O}(a)$ ambiguities. These ambiguities only affect $P_{5}$-even quantities at $\mathrm{O}\left(a^{2}\right)$ [5]. On the other hand, the simultaneous determination of $m_{\mathrm{cr}}$ and $z_{f}$ can become difficult if these $\mathrm{O}(a)$ effects are large, since $m_{\mathrm{cr}}$ could depend strongly on $z_{f}$ in this case. We noticed however that once the bulk action is improved, $m_{\mathrm{cr}}$ is basically independent from $z_{f}$ over a wide range of values around the target one. The tuning is then straightforward. This confirms what was observed in quenched studies $[9,10]$.

To conclude, in the following we focus on the set of bare couplings $g_{0}$ defined by $\beta=6 / g_{0}^{2} \in$ $\{5.2,5.3,5.5,5.7\}$. The corresponding lattice resolutions $(L / a)\left(g_{0}\right)$ were then chosen such that $L=0.6 \mathrm{fm}$, within a few per cent. The resulting lattice sizes are given by $L / a=8,9.2,10,12,16$. Note that, the results at $L / a=9.2$ were obtained from an interpolation of the results of three lattices with $L / a=8,10,12$, and fixed $\beta=5.3$.

\section{Determination of $Z_{A}$ and $Z_{V}$}

In Figure 1, we present the results for the non-singlet axial and vector current renormalizations, $Z_{A}$ and $Z_{V}$. We show the results corresponding to the $\chi$ SF definitions (2.4), together with the standard SF determinations, $Z_{A}^{\mathrm{SF}}$ and $Z_{V}^{\mathrm{SF}}$. ${ }^{1}$ As we can see from the plot, the $\chi \mathrm{SF}$ results are nicely consistent with the SF determinations. Agreement is generally found within errors. This indicates that the $\mathrm{O}\left(a^{2}\right)$ differences between the SF and $\chi \mathrm{SF}$ determinations are in general much smaller than the errors on the standard SF values. In fact, due to the much higher precision of the $\chi \mathrm{SF}$ determinations, we can appreciate some difference between the different definitions of $Z_{A, V}$. These $\mathrm{O}\left(a^{2}\right)$ effects are small, and at most a couple of per cent at the largest value of $g_{0}$ (cf. Figure 2).

To conclude, in Table 1 we collected the preliminary results for $Z_{A, V}$ as obtained from the $\chi \mathrm{SF}$. Note that the errors include an estimate of the systematic uncertainties in $Z_{A, V}$ due to the finite precision with which we satisfied the conditions: $m_{\mathrm{PCAC}}=0, g_{A}^{u d}(T / 2)=0$, and $L=0.6 \mathrm{fm}$.

\footnotetext{
${ }^{1}$ For $Z_{A}^{\mathrm{SF}}$ we used the interpolation formula (B.1) in [13], while for $Z_{V}^{\mathrm{SF}}$ we took the results in Table 1 of [14].
} 

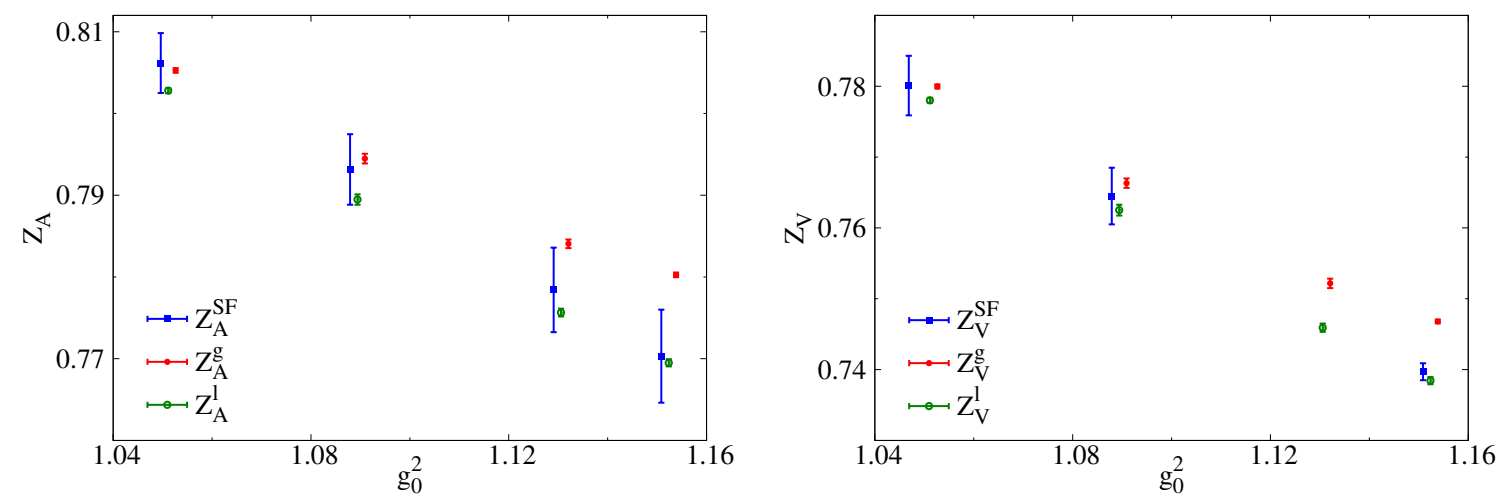

Figure 1: Results from the $\chi \mathrm{SF}$ and standard SF for the finite renormalization constants $Z_{A}$ and $Z_{V}$. The points have been slightly shifted in $g_{0}^{2}$ in order to improve the readability of the plot.

\begin{tabular}{ccccc}
\hline$\beta$ & $Z_{V}^{g}$ & $Z_{V}^{l}$ & $Z_{A}^{g}$ & $Z_{A}^{l}$ \\
\hline 5.2 & $0.74680(26)$ & $0.73844(51)$ & $0.78026(28)$ & $0.76950(45)$ \\
5.3 & $0.75217(67)$ & $0.74592(59)$ & $0.78406(52)$ & $0.77564(48)$ \\
5.5 & $0.76632(67)$ & $0.76251(76)$ & $0.79448(59)$ & $0.78948(64)$ \\
5.7 & $0.77997(29)$ & $0.77801(36)$ & $0.80527(29)$ & $0.80280(32)$ \\
\hline
\end{tabular}

Table 1: Preliminary results for $Z_{A}$ and $Z_{V}$ as obtained from the $\chi \mathrm{SF}$.

\section{Automatic $\mathrm{O}(a)$ improvement}

We now address the issue whether automatic $\mathrm{O}(a)$ improvement is at work. In this respect in Figure 2 (left panel), we present the approach to the continuum limit of the difference between different definitions of $Z_{A, V}$ (cf. (2.4)). As we can see from the plot, the scaling to the continuum limit is nicely $\mathrm{O}\left(a^{2}\right)$ for the lattices considered; the lines on the plot are linear fits in $(a / L)^{2}$ constrained to zero. This is a clear indication for $\mathrm{O}(a)$ improvement being automatic. We note in fact that even though the bulk action is improved, the full $\mathrm{O}(a)$ improvement of these determinations would otherwise require the improvement of the corresponding bulk operators entering in the definitions. This is further corroborated by considering the improved axial current $\left(A_{I}\right)_{\mu} \equiv A_{\mu}+c_{A}\left(g_{0}\right) a \tilde{\partial}_{\mu} P$ [14] in the definition of $Z_{A}^{g}$, thus introducing, $Z_{A_{I}}^{g}$. As we can see from the figure, the difference between these two definitions is compatible with zero. We interpret this as the fact that the $\mathrm{O}(a)$ counterterm for the axial current contributes only at $\mathrm{O}\left(a^{2}\right)$, and that this effect is negligible within errors.

The complementary feature of automatic $\mathrm{O}(a)$ improvement is that $P_{5}$-odd correlators are pure $\mathrm{O}(a)$ lattice artifacts. In Figure 2 (right panel), we present the continuum limit of several $P_{5}$-odd correlators (cf. (2.3)). As we can see from the plot, all correlators but $l_{\tilde{V}}^{u d}$ are compatible with zero for the lattice resolutions considered. The $l_{\widetilde{V}}^{u d}$ correlator then vanishes with the expected $\mathrm{O}(a)$ scaling, as illustrated by a linear fit in $a / L$ constrained to zero. Note that the sizable $\mathrm{O}(a)$ effects in $l_{\widetilde{V}}^{u d}$ are due to the $\mathrm{O}(a)$ operator counterterm of $\widetilde{V}_{\mu}$. Indeed, if we consider the improved definition, $\left(\widetilde{V}_{I}\right)_{\mu} \equiv \widetilde{V}_{\mu}+c_{\widetilde{V}}\left(g_{0}\right) a \tilde{\partial}_{v} T_{\mu \nu}$, and use the tree-level value, $c_{\widetilde{V}}=\frac{1}{2}$, we see that cutoff effects are significantly reduced in $l_{\widetilde{V}_{I}}^{u d}$ compared to $l_{\widetilde{V}}^{u d}$. 

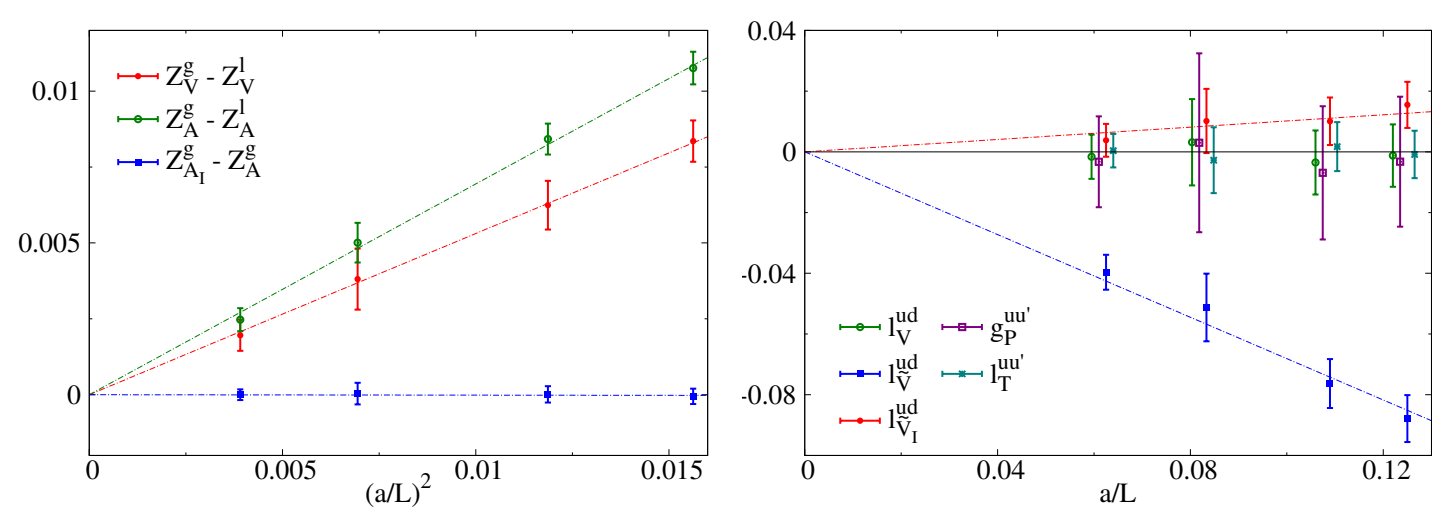

Figure 2: Left: Continuum limit extrapolations for differences of different definitions of $Z_{A, V}$. Right: Continuum limit extrapolations for several $P_{5}$-odd correlators. Some of the points are shifted in $a / L$ in order to improve the readability of the plot. The continuum limit is reached as $a / L \rightarrow 0$.

\section{Renormalization of the pseudo-scalar density}

In this section we present some results for the renormalization of the pseudo-scalar density. The renormalization condition we consider, and corresponding step-scaling function are given by,

$$
Z_{P}^{\chi \mathrm{SF}}\left(g_{0}, L / a\right)=\left.c(L / a) \frac{\sqrt{3 g_{1}^{u d}}}{g_{P}^{u d}\left(x_{0}\right)}\right|_{x_{0}=\frac{T}{2}}, \quad \Sigma_{P}^{\chi \mathrm{SF}}(u, a / L)=\left.\frac{Z_{P}^{\chi \mathrm{SF}}\left(g_{0}, 2 L / a\right)}{Z_{P}^{\chi \mathrm{SF}}\left(g_{0}, L / a\right)}\right|_{u=\bar{g}^{2}(L)},
$$

where the constant $c$ is chosen such that $Z_{P}^{\chi \mathrm{SF}}(0, L / a)=1$, while $\bar{g}^{2}(L)$ is a given finite-volume coupling. The standard SF definition, $Z_{P}^{\mathrm{SF}}$, is analogously defined in terms of the corresponding SF correlators (cf. (2.2)). We refer to [15] for the details and the SF results used in this section.

In the left panel of Figure 3, we look at the ratio $Z_{P}^{\chi \mathrm{SF}} / Z_{P}^{\mathrm{SF}}$ which should approach 1 in the continuum limit with $\mathrm{O}\left(a^{2}\right)$ corrections. Note that $L$ is kept fixed in terms of the finite-volume coupling $\bar{g}^{2}(L)$, and we consider three lattice-sizes $L / a=6,8,12$, for the continuum extrapolations. As we can see from the plot, the differences between the SF and $\chi \mathrm{SF}$ results are quite small, and in fact below the per cent for all three values of $\bar{g}^{2}(L)$ considered, even at the smallest resolution $L / a$.

Similar conclusions can be drawn from the right panel of Figure 3, where we computed the continuum limit extrapolation of the corresponding step-scaling functions at the largest value of $\bar{g}^{2}(L)$. As we can see from the figure, the continuum values for the SF and $\chi$ SF nicely agree.

\section{Conclusions}

In this contribution we have presented first results from dynamical simulations of the chirally rotated Schrödinger functional of QCD. At the little extra cost of renormalizing the boundary conditions, the set-up offers competitive methods for the determination of finite renormalization constants, and it is compatible with automatic $\mathrm{O}(a)$ improvement. This makes it an interesting alternative to consider for the renormalization of complicated operators like for example 4-quark operators, and it is a natural framework to solve renormalization problems in twisted-mass lattice QCD at maximal twist. 

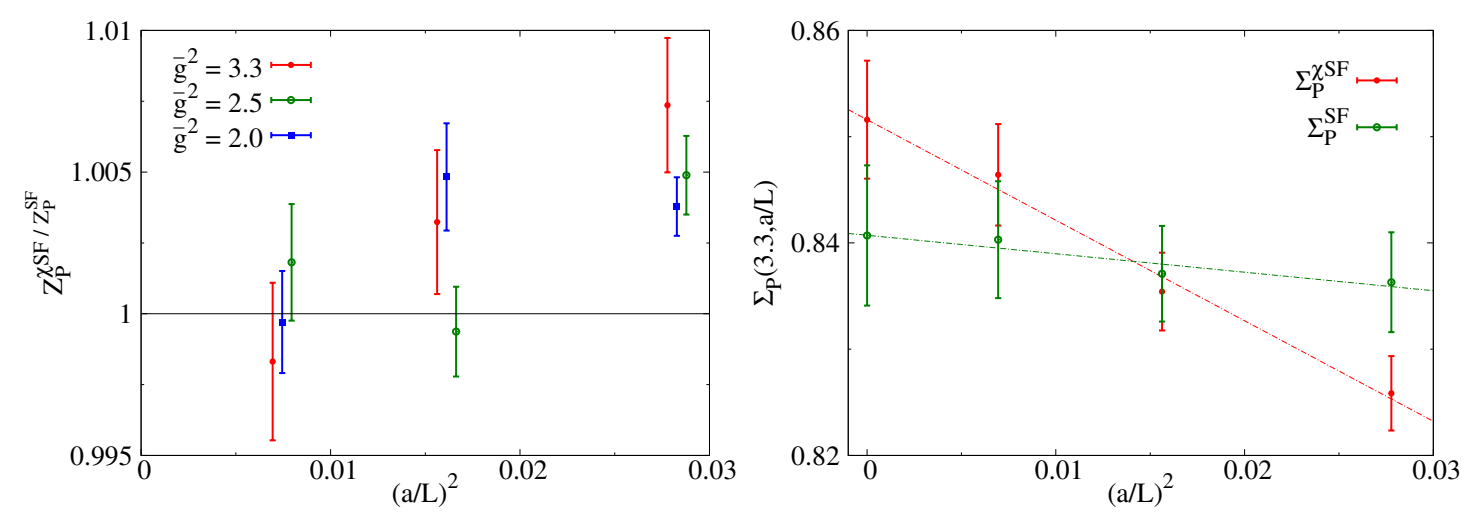

Figure 3: Left: Comparison between the renormalization of the pseudo-scalar density as computed with the SF, $Z_{P}^{\mathrm{SF}}$, and $\chi \mathrm{SF}, Z_{P}^{\chi \mathrm{SF}}$. Right: Continuum limit extrapolation for the corresponding step-scaling functions at $\bar{g}^{2}=3.3$. Note that in [15] a constant fit was considered for the SF results.

\section{Acknowledgments}

The authors warmly thank J. Bulava for his contribution at an early stage of this project. We also thank S. Lottini and R. Sommer for discussions, and S. Schaefer for many valuable comments. M.D.B. is funded by the Irish Research Council. S. Sint acknowledges support by SFI under grant 11/RFP/PHY3218. The computer resources provided by TCHCP and ICHEC are also gratefully acknowledged. The simulation code we used is a customized version of the openQCD code of [16].

\section{References}

[1] M. Lüscher, R. Narayanan, P. Weisz, and U. Wolff, Nucl. Phys. B384 (1992) 168-228.

[2] S. Sint, Nucl. Phys. B421 (1994) 135-158.

[3] R. Frezzotti and G. Rossi, JHEP 0408 (2004) 007.

[4] S. Sint, PoS LATTICE2005 (2006) 235.

[5] S. Sint, Nucl. Phys. B847 (2011) 491-531.

[6] R. Frezzotti, P. A. Grassi, S. Sint, and P. Weisz, JHEP 0108 (2001) 058.

[7] S. Sint and P. Vilaseca, PoS LATTICE2012 (2012) 031.

[8] S. Sint and P. Vilaseca, PoS LATTICE2014 (2014) 279.

[9] S. Sint and B. Leder, PoS LATTICE2010 (2010) 265.

[10] J. G. Lopez, K. Jansen, D. Renner, and A. Shindler, Nucl.Phys. B867 (2013) 567-608.

[11] J. G. Lopez, K. Jansen, D. Renner, and A. Shindler, Nucl.Phys. B867 (2013) 609-635.

[12] S. Sint and P. Weisz, Nucl. Phys. B502 (1997) 251-268.

[13] P. Fritzsch, et al., Nucl.Phys. $\mathbf{B 8 6 5}$ (2012) 397-429.

[14] M. Della Morte, et al., JHEP 0507 (2005) 007.

[15] M. Della Morte, et al., Nucl. Phys. B729 (2005) 117-134.

[16] M. Lüscher and S. Schaefer, Comput. Phys. Commun. 184 (2013) 519-528. 